\title{
Contaminación visual en centros históricos: un problema estético y de valor social de las ciudades actuales
}

\author{
Visual pollution in historical centers: an aesthetic problem and of social value of the \\ present cities
}

\author{
Angélica Sierra Franco, ${ }^{1}$ Esp; Oscar Guevara Guevara, ${ }^{2}$ Esp. \\ ${ }^{1}$ Corporación Universitaria del Caribe - CECAR, Programa de Arquitectura, Sincelejo, Colombia. \\ Correspondencia: angelica.sierraf@cecar.edu.co \\ ${ }^{2}$ Corporación Universitaria del Caribe - CECAR, Programa de Arquitectura, Sincelejo, Colombia. \\ Correspondencia: oscar.guevara@cecar.edu.co
}

Recibido: 15 de agosto de 2016. Aceptado: 22 de octubre de 2016.

Sierra, A.; Guevara, O. (2016) Contaminación visual en centros históricos: un problema estético y de valor social de las ciudades actuales. Procesos urbanos Número 3, Ene-Dic. 133-142. Doi:10.21892/2422085X.273

\section{RESUMEN}

La contaminación visual en los centros urbanos debe ser considerada en nuestros tiempos como un factor determinante en el deterioro de la calidad de vida y la ruptura del paisaje urbano, en tanto provoca una desvalorización de la propiedad pública y privada, como resultado de la dinámica desordenada de la competitividad en las dimensiones institucionales, cívicas, políticas y comerciales. Por tanto, debe ser regulada, prevenida y controlada. Este planteamiento surge de la necesidad de promover la conservación, protección y sostenibilidad del paisaje urbano, conformado por los elementos que constituyen el espacio público del centro de Sincelejo, y su valoración histórico-patrimonial, como factor fundamental para preservar y mantener el equilibrio visual de los mismos. El trabajo tiene como finalidad garantizar el derecho constitucional de gozar de un ambiente sano y ecológicamente equilibrado. Y que, a su vez, se convierta en un eje articulador de las propuestas turísticas que tiene la región, en cuanto a la promoción de los bienes inmuebles culturales e históricos que se emplazan en esta zona.

Palabras clave: Contaminación visual, centros históricos, entornos urbanos, ambiente, percepción ciudadana, actores

\section{ABSTRACT}

The visual pollution in urban centers must be considered in our times as a determining factor in the deterioration of the quality of life and the rupture of the urban landscape, while causing a devaluation of public and private property, because of the disordered dynamics of competitiveness in the institutional, civic, political and commercial dimensions. Therefore, it must be regulated, prevented and controlled. This approach arises from the need to promote the conservation, protection and sustainability of the urban landscape, made up of the elements that constitute the public space of the center of Sincelejo, and its historical and patrimonial valuation, as a fundamental factor to preserve and maintain the visual balance of the same. The purpose of the work is to guarantee the constitutional right to enjoy a healthy 
and ecologically balanced environment. Moreover, that, in turn, become an articulating axis of the tourist proposals that the region has, in terms of the promotion of cultural and historical real estate that are located in this area.

Keywords: Visual pollution, historical centers, urban environments, environment, citizen perception, actors

\section{INTRODUCCIÓN}

La acción del hombre en el entorno genera transformaciones positivas o negativas; estas alteraciones tienen repercusiones tanto en el entorno, como en el hombre, en una dialógica urbana de causas y efectos.

Muchas ciudades, enviciadas por el consumo, dejan a un lado sus características de sistema territorial urbano-rural que las identifica y entran en la tan anhelada modernización, convirtiéndose en una fuente de inquietud y desarraigo, dominada por la irracionalidad de los contrastes y la crueldad de las desigualdades. A los centros urbanos, que también son los lugares de la emancipación, de la acumulación de las riquezas, de la iniciativa individual y de la creación, se les añaden las grandes preocupaciones ambientales amenazadoras, problemas que se ven acompañados por la pérdida de ciudadanía, por la anomia social y la degradación cultural.

Los entornos urbanos, en especial los centros de ciudad, han sido los contenedores ${ }^{1}$ de todos los procesos de crecimiento territorial y poblacional; las constantes migraciones forzadas y voluntarias que reciben las ciudades, exigen una acelerada transformación de las mismas, y es entonces, cuando la dialógica correlacional entra en acción, generando un acelerado y continuo deterioro, producto de las cargas a las que es sometido el ambiente.

Por un lado, los centros urbanos afrontan los problemas típicos de las ciudades actuales, engendradas por una sociedad moderna y capitalista, en la era del consumo. Por otro lado, se encuentran los lugares de encuentro, de sociabilidad, de ley y de fe que difunden una ética ciudadana. Producto de estas dos versiones sociales de la ciudad, se trazan

1 Se le dice contenedor a la caja de carga que cumple la función de tener dentro de sí alguna otra cosa. los antagonismos y las contradicciones que viven los centros urbanos hoy.

Los procesos de contaminación, en especial aquellos que afectan la calidad visual del paisaje urbano (Díaz, 1997), para las ciudades cuya centralidad guarda importantes elementos históricos de memoria colectiva y bienes inmuebles de valor patrimonial, se convierten en un problema de valor estético y social que ha preocupado a muchos a lo largo y ancho del planeta y que ha suscitado la imposición de estrictas normativas y movimientos de resistencia social en defensa del patrimonio.

El tema de la estética urbana y de la calidad visual, no solo se viene tratando como un problema ambiental y de salud pública, sino, que repercute en las esferas gubernamentales y la legitimidad de los espacios como eje fundamental para hacer ciudades amables y turísticamente atractivas y competitivas.

La contaminación visual urbana, especialmente en las ciudades intermedias de países en vía de desarrollo, es uno de los factores más nocivos que alteran el equilibrio ambiental, como resultado de la dinámica desordenada de la competitividad en las dimensiones institucionales, cívicas, políticas y comerciales.

La ciudad se expande y con ella el comercio, los servicios y los desechos del consumismo; el aparato productor necesita exponer sus mercancías y lo hace a través de vallas, anuncios, pancartas y volantes que, sin ningún tipo de organización entran a ser parte de un alterado panorama urbano, al que se le adicionan un mal manejo de elementos como basuras, cableado eléctrico, grafitis, comercio informal y por último, el deterioro físico de las edificaciones por falta de mantenimiento. 
Este hecho, vivenciado y percibido por los habitantes del territorio, trae como consecuencias el detrimento de la calidad de vida y la ruptura del paisaje, en tanto provoca una desvalorización de la propiedad pública y privada, pérdida de valor escénico y paisajístico de la zona, falta de apropiación de los centros urbanos en la identificación de espacios de legitimidad ciudadana, alteraciones del estado emocional y físico de los individuos, es además impulsora de malas conductas ciudadanas debido a que hace que el habitante perciba una ciudad en degradación, pobreza y abandono, que repercuten en el potencial turístico y económico del lugar.

La ciudad de Sincelejo, capital del departamento de Sucre en la República de Colombia, lugar en donde se desarrolla la presente investigación, se presenta como una ciudad intermedia, con características urbano-rurales y polo de concentración de gran parte de la población desplazada de los Montes de María, el Cauca y Antioquia en el trascurso del periodo violento del país. La rápida metamorfosis de este territorio pone en evidencia el acelerado impacto al ambiente por causa de este contaminante, que se convierte en el medio de expresión y subsistencia de una sociedad desorganizada y carente de organización, lo que facilita la acumulación de los elementos mencionados anteriormente que afectan el paisaje urbano.

La caracterización de la contaminación visual en el espacio público de la ciudad de Sincelejo, determinó la influencia que tienen los impactos visuales en la imagen urbana y la percepción de la ciudad por parte de los ciudadanos y visitantes; esta información permitió generar una evaluación de los elementos contaminantes, sus actores y en qué nivel de saturación se encuentran, convirtiéndose en un soporte para el planteamiento de recomendaciones y alternativas que permitan implementar medidas de control y regulación, no solo jurídicas, mediante la aplicación de la norma local vigente sino, con la incorporación de elementos de diseño urbano con parámetros claves demanejo del color, dimensionamiento, materialidad y propuestas pedagógicas de participación ciudadana.

\section{El concepto de contaminación visual.}

Los centros urbanos acogen en su interior las dinámicas de la población en su constante diálogo con el medio físico, sea natural o arquitectónico, que trae como consecuencias del intercambio, muchas veces un influjo de desechos urbanos, a los que se denominan contaminantes.

El contaminante que afecta el sentido visual del habitante en la percepción de la estética de su entorno, ha sido tema de disertación de numerosas investigaciones que definen el concepto de contaminación visual como "abuso de ciertos elementos -no arquitectónicos-, que alteran la estética, la imagen del paisaje rural o urbano" (Rozada, 2006; En, Méndez, 2013). Estas alteraciones estéticas son igualmente definidas por Hess (2006), como "cambio o desequilibrio en el paisaje, ya sea natural o artificial que afecta las condiciones de vida y las funciones vitales de los seres vivientes" (Hess, 2006; En, Méndez, 2013).

Ambasdefiniciones, exponencoherentemente las causas y los efectos de la contaminación visual, de manera que, el estudio de esta problemática es de vital importancia para resguardar no solo la imagen de las ciudades, sino el libre desarrollo de sus habitantes, su comportamiento y modos de vida.

Por otro lado, algunas publicaciones en el campo del urbanismo y la arquitectura destacan el efecto letal que tiene la contaminación para el escenario urbano ya que contribuye a erosionar la humanidad de las ciudades.

Esta última afirmación, pone de manifiesto, que si bien la contaminación visual trae consecuencias sociales, además de las estéticas, uno de los mayores agentes contaminantes también es la misma sociedad, convirtiéndose este hecho en un círculo vicioso de degradación territorial y humana, que induce a la reflexión de que las crisis de las ciudades, son a su vez crisis sociales. 
Un punto de vista valioso para contrarrestar la problemática de la contaminación visual en las ciudades es el brindado por García (2013), quien reflexiona al respecto de la siguiente manera:

La contaminación visual urbana es un fenómeno de polución de características singulares, debido a que no se transmite a través de un vector sobre el que se pueda actuar. Por lo tanto, el trabajo de las autoridades debe orientarse principalmente hacia su origen, es decir, la descontrolada proliferación de publicidad. Es por ello que la solución a esta problemática debe basarse, principalmente, en un adecuado marco normativo y en un eficiente ejercicio del poder de la policía municipal que tienda a proteger a los habitantes y el patrimonio arquitectónico de los indiscriminados ataques de la cultura del consumismo y la competencia por el deprimido mercado local, que se manifiesta en una superposición permanente de anuncios de dudosa legalidad y en muchos casos, de un igualmente dudoso gusto. (p.4)

\section{Agentes y actores de la contaminación visual.}

Los agentes contaminantes del espacio público en su calidad visual, adquieren sus características según el propósito con el que los actores de la contaminación los exponen y propagan.

La mayoría de autores que exponen en esta temática, coinciden en identificar los contaminantes visuales, según la siguiente enumeración:

Carteles publicitarios (vallas, avisos luminosos, pancartas, pinturas, carteles, entre otros).

Grafitis.

Antenas.

Cableado aéreo.

Condensadores de aires acondicionados sobre las fachadas.

Mezcla de estilos arquitectónicos.

Basurales.

Árboles en incorrecta disposición.

Deterioro de las fachadas.
Estos elementos, por sí solos o en conjunto, causan una saturación del espacio público que dificulta la lectura del paisaje. El manejo abusivo de los mismos por parte del hombre y la falta de planeación urbana, generan en la percepción del ciudadano una sobre estimulación visual excesiva, agresiva e indiscriminada, que no solo termina con deteriorar la imagen de la ciudad, sino también, apropiación ciudadana.

Son muchas las acciones sobre la ciudad que se convierten finalmente en contaminación. Gámez (2013), afirma que se constituyen como foco de contaminación visual las estrategias implementadas por las campañas políticas al ubicarse en lugares indebidos, lo cual genera saturación visual. Esta situación es sensiblemente nociva en centros históricos debido a la existencia en esas zonas de edificios de alto valor patrimonial.

\section{Percepción y afectación de la contaminación visual.}

"La percepción es la puerta por la que el mundo entra a la mente humana, pero también, la ventana por la que la mente sale al mundo" (Fernández, 2003).

La percepción humana implica la capacidad consciente de habitar un mundo objetivo y compartido. Según Rapoport (1974), la percepción es el mecanismo más importante que relaciona a los hombres con su ambiente. En este proceso, los sentidos son los órganos físicos y psicológicos facultados para acometer esta acción, y la vista, es sin duda, el órgano que pone en evidencia el entorno ante el hombre, permitiéndole generar juicios de valor en cuanto a la calidad del entorno apreciado.

La percepción visual necesariamente involucra al que percibe y al medio percibido, contribuye en gran medida a la orientación, y se apoya en el espacio, la distancia, textura, color, forma, contraste, entre otros (Méndez, 2013).

Los estímulos percibidos, son seleccionados y procesados consciente o inconscientemente. La información percibida que no es seleccionada y procesada, se guarda en el 
inconsciente, lo que afecta a las personas emocional y físicamente.

Estas afectaciones van desde problemas de salud física como mareos, tensión y dolor de cabeza, hasta situaciones actitudinales que influyen directamente en la apropiación y sentido de pertenencia con los lugares estimuladores de estos impactos. Nasar (1994) ha investigado, cómo la estética del exterior de las edificaciones, afecta las respuestas evaluativas y afectivas de las personas ante el paisaje urbano (Méndez, 2013).

Esta situación genera grandes inconvenientes urbanos, puesto que sumado al deterioro físico causado por la contaminación visual, la crisis ciudadana de desaprobación y desarraigo por los lugares contaminados visualmente, que no brindan comodidad y bienestar físico, generan una desconexión con la ciudad y una falta de responsabilidad con la imagen de la misma.

Un espacio urbano poco legible es difícilmente legitimizable, por cuanto dificulta la identificación del habitante con su hábitat, del ciudadano con su ciudad; es un espacio deteriorado no solo estéticamente sino, socialmente. Es un espacio deshumanizado.

\section{CONTAMINACIÓN VISUAL EN LA CIUDAD DE SINCELEJO}

\section{Problemática actual}

La problemática actual de contaminación visual que afronta el espacio público del centro histórico de Sincelejo, fue abordada desde diferentes aspectos, dentro de ellos se encuentra, la identificación de los tipos de contaminación visual, la cual se aprecia en la figura 1.

En la anterior gráfica se observa que el principal tipo de contaminación visual presente en la zona de estudio son las redes del cableado eléctrico, internet, telefonía y postes de luz, con un porcentaje del $31 \%$. Ver figura 2 .

En ese orden de ideas, el segundo tipo de contaminante visual encontrado en el espacio público del centro histórico de Sincelejo es la superposición de estilos arquitectónicos con un porcentaje del $25 \%$.

Asimismo, el tercer tipo de contaminante visual detectado es el generado por el inadecuado diseño y disposición de avisos

\section{TIPOS DE CONTAMINACION PREDOMINANTES ENEL}

\section{CENTRO DE SINCELEJO}

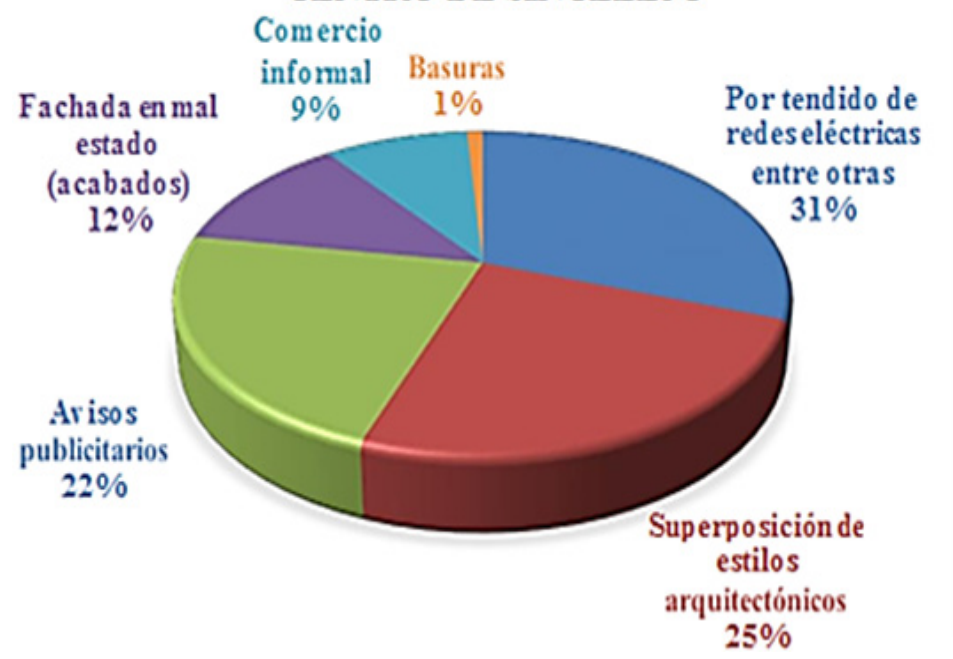

Figura 1. Tipos de contaminación visual en el espacio público del centro histórico de Sincelejo. 

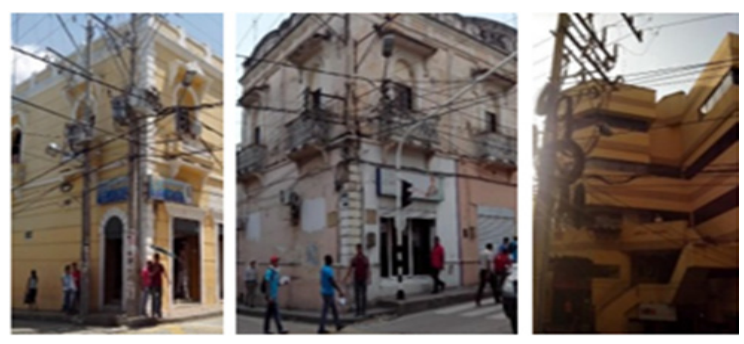

Figura 2. Contaminación visual por redes de cableado eléctrico.

Fuente de elaboración: propia.

publicitarios. En ese sentido, se estima un porcentaje del $22 \%$ para la contaminación visual generada por avisos publicitarios. Ver figura 3.

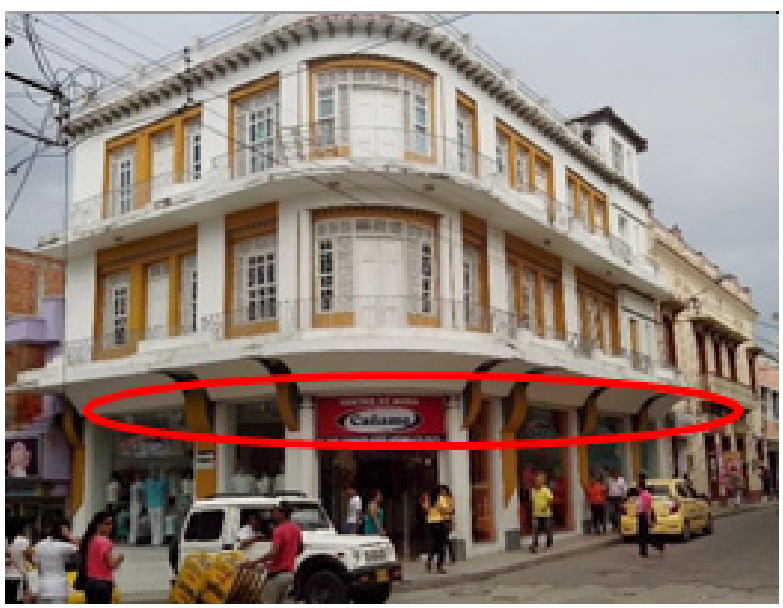

Figura 3. Contaminación visual por avisos publicitarios. Fuente de elaboración: propia.

Al continuar con la identificación de los tipos de contaminantes visuales, se aprecia que el cuarto tipo de contaminante visual en el espacio público del centro histórico de Sincelejo es el generado por las fachadas de edificaciones en mal estado. Por lo tanto, el porcentaje de contaminación visual por fachadas en mal estado se estimó en un $12 \%$. Ver figura 4.

El quinto lugar de contaminantes visuales identificados lo ocupa el generado por el comercio informal, el cual presenta un porcentaje del $9 \%$.

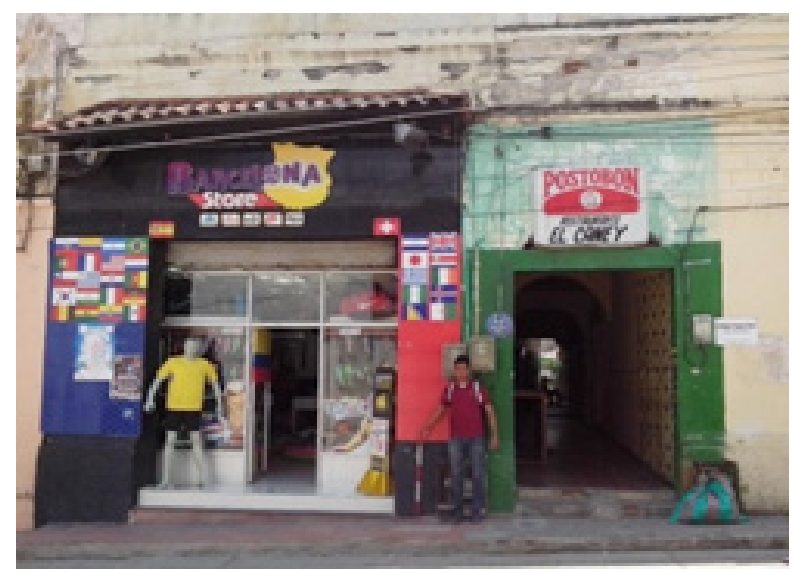

Figura 4. Contaminación visual por fachadas de edificaciones en mal estado.

Fuente de elaboración: propia.

En última instancia se identificó el contaminante visual producto de la indebida disposición de basuras con un porcentaje del $1 \%$. Ver figura 5.

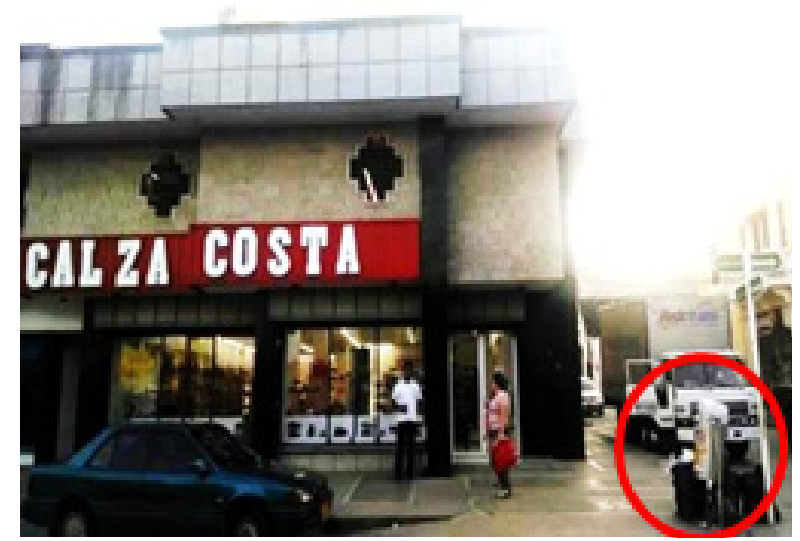

Figura 5. Contaminación visual por disposición indebida de basuras.

Fuente de elaboración: propia

Dentro de los aspectos tenidos en cuenta para abordar el fenómeno de la contaminación visual, fue trascendental establecer el porcentaje de fachadas de edificaciones con contaminación visual, versus el porcentaje de fachadas de edificaciones sin contaminación visual. En ese orden de ideas, se estimó que el área total de las fachadas, de las 351 edificaciones levantadas, es de $=46407,46$ $\mathrm{m} 2$. Además, el área total de las fachadas, sin contaminación visual, es equivalentes al $38 \%$, versus el área total de las fachadas, con contaminación visual, son equivalentes al $62 \%$. Figura 6. 
GRÄFICA DEL PORCENTAJE DE FACHADAS CON CONTAAIINACION VISUAL YS PROCENTAJE DE FACHADAS SIN CONTAMINACIÓX VISUAL.

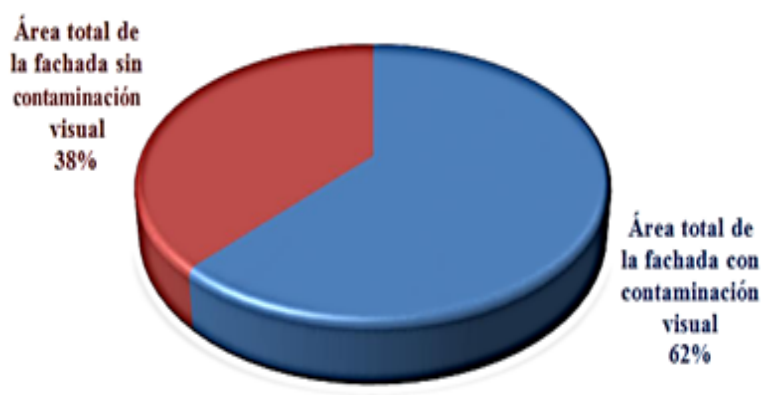

Figura 6. Porcentaje de fachadas con contaminación visual en el centro de Sincelejo.

Fuente de elaboración: propia.

De acuerdo con lo anterior, se establece que en las fachadas, es mayor el área contaminada por elementos visuales que el área que no posee este tipo de contaminantes, ya que el área de fachadas con contaminantes visuales es de $28772,62 \mathrm{~m} 2$ frente al área de fachadas sin contaminantes visuales la cual es de $17634,83 \mathrm{~m} 2$.

\section{Percepción ciudadana}

El estado de la percepción ciudadana frente al fenómeno de la contaminación visual, fue identificado por medio de una encuesta de percepción que se aplicó a la ciudadanía, lo cual permitió transcender de aspectos cuantitativos a aspectos cualitativos en la investigación. Declarando que el $70 \%$ de la ciudadanía encuestada no saber si existe una normativa que regule la contaminación visual en la ciudad de Sincelejo. Y solo el $5 \%$ de la población encuestada, opinó que sí conoce la normativa existente. Por lo tanto, el $25 \%$ de la población restante respondió que no existe una normativa que maneje los niveles de contaminación visual en esta ciudad.

\section{Antecedentes legales}

En Sincelejo, en el año 2008 se adoptan mediante el Acuerdo 28 de 2008, políticas para el manejo integral y el uso del espacio público y la publicidad exterior visual en la ciudad, que en su artículo 1, tiene como objeto "mejorar la calidad de vida de los ciudadanos residentes en el municipio, en consonancia con los derechos a la comunicación, al medio ambiente sano, la protección de la integridad del espacio público y la seguridad vial".

Seguidamente en el artículo 2, nos presentan como definición del espacio público:

El elemento articulado y estructurante fundamental del espacio en la ciudad, así como el regulador de las condiciones ambientales de la misma, y por tanto se constituye en uno de los principales elementos estructurales de los planes de ordenamiento territorial.

Y según el artículo 5 de publicidad exterior visual:

El medio masivo de comunicación, permanente o temporal, fijo o móvil, que se destine a llamar la atención del público a través de leyendas o elementos visuales en general, tales como inscripciones, dibujos, fotografías, signos o cualquier otra forma de imagen que se haga visible desde las vías de uso o dominio público, bien sean peatonales o vehiculares, terrestres, acuáticas o aéreas y cuyo uso sea comercial, cívico, cultural, político, institucional e informativo. Tales pueden ser vallas, avisos, tableros electrónicos, pasacalles, pendones, colombinas, carteleras, mogadores, globos y otros similares.

En el artículo 6, se reglamentan las prohibiciones generales, donde no podrá colocarse publicidad exterior visual en:

Las áreas que constituyan espacio público de conformidad con las normas municipales, exceptuando las fachadas de los inmuebles y aquellas que contengan mensajes de tipo institucional.

Las zonas históricas, edificios o sedes de entidades públicas y embajadas, salvo que se trate de los avisos que indican el nombre de las entidades, embajadas y lugares históricos y de la publicidad exterior visual que de manera eventual anuncie obras de remoción o eventos artísticos. 
En los sectores residenciales no podrá tener iluminación, salvo que se trate de avisos adosados a la pared de establecimientos comerciales.

En lugares en los que su colocación obstaculice el tránsito peatonal, en donde interfiera con la visibilidad de la señalización vial, informativa y de la nomenclatura urbana, aun cuando sean removibles.

Según el artículo 7, se define como AVISO: El elemento que se utiliza como anuncio, identificación, señal, advertencia o propaganda con fines profesionales, culturales, comerciales, turísticos o informativos se instala adosado a las fachadas de edificaciones de manera permanente.

Aclarando en el artículo 8 que:

Sólo podrá existir un aviso por fachada. Los avisos no podrán exceder el $30 \%$ del área de la fachada del primer piso del respectivo establecimiento, no podrá utilizar áreas o elementos complementarios de fachada, ni estará su borde inferior a una altura menor de 2.10 metros sobre el nivel del andén. En cualquier caso, los avisos no podrán tener un área superior a 15 metros cuadrados.

Cuando las características del edificio o del sector no permitan la colocación del aviso en la fachada del primer piso, se permitirá su colocación en el antepecho del segundo. Para el caso de los inmuebles definidos como de patrimonio, el tamaño de estos avisos debe ser de $0.6 \mathrm{~m}$. de alto por 2 metros de largo, debe ser de material de madera con letras de bronce.

En el centro histórico de Sincelejo, los avisos no podrán superar los 4 metros cuadrados.

Los avisos ubicados en las zonas residenciales tendrán un área máxima o inferior al 10\% del área de la fachada del primer piso del establecimiento.

Cuando en una misma edificación se desarrollen varias actividades comerciales, estas se anunciarán dentro de un mismo marco, el cual deberá cumplir con los requerimientos de este acuerdo.
En el artículo 9, tiene como prohibiciones. No colocar avisos bajo las siguientes condiciones:

Los avisos volados o salientes de la fachada. Los pintados o incorporados en cualquier forma a las ventanas o puertas de la edificación.

Los adosados o suspendidos en antepechos superiores al segundo piso.

Cuando utilicen forma de signo o señal de tránsito con fines publicitarios.

Queda claro en el artículo 10, que son responsables por el incumplimiento, las personas naturales o dueñas del aviso o el propietario del establecimiento que coloque o permita colocar los avisos sin el cumplimiento de los requisitos.

Según el artículo 11, se define como VALLA: Entiéndase porvalla todo anuncio permanente o temporal utilizado como medio masivo de comunicación, que permite difundir mensajes publicitarios, cívicos, comerciales, turísticos, políticos, institucionales, artísticos, informativos o similares, que se colocan para su apreciación visual en lugares exteriores y que se encuentra montado sobre una estructura metálica u otro material estable con sistemas fijos, el cual se integra física, visual, arquitectónica y estructuralmente al elemento que lo soporta.

Según el artículo 12, se tiene que, por su ubicación:

Las vallas en el municipio de Sincelejo podrán ubicarse sobre corredores de actividad múltiple, teniendo en cuenta que su distancia mínima en el mismo sentido y costado vehicular, no podrá ser inferior a cien metros (100m.) y en las condiciones previstas a continuación:

En los lotes privados construidos y sin construir, se podrán colocar vallas convencionales y tubulares, siempre y cuando su dimensión no supere los cuarenta y ocho metros cuadrados (48m.).

En obras en construcción, restauración, remodelación, adecuación o ampliación: 
En estos casos solo podrán colocarse dos 2 vallas, las que deberán instalarse antes de iniciar la obra y retiradas hasta seis (6) meses después de haberse terminado la misma. Se ubicarán del parámetro del predio adentro y contendrán la información solicitada por la Secretaria de Planeación Municipal. No podrán ocupar más $10 \%$ del área de la fachada.

En las culatas de las edificaciones: siempre y cuando el área de la valla no exceda en setenta $(70 \%)$ del área de la culata, ni 48 $\mathrm{m}^{2}$.

En cubiertas de las edificaciones: Se podrán instalar con una altura máxima de 4 metros sobre el nivel de la construcción permitido en el sector.

En los patios internos y parqueaderos: Únicamente cuando la estructura sea tubular, la altura máxima no supere los 24 metros y los vértices de la valla no superen por ninguno de los costados los límites del inmueble.

Al momento de colocar la publicidad exterior visual se debe registrar con (3) tres días de anticipación a su colocación. El cual debe llevar tipo de publicidad y ubicación, identificación del anunciante, NIT, ilustración o fotografía de la publicidad exterior visual y transcripción del texto que allí va a aparecer.

Si la publicidad no aplica los lineamientos de esta norma vigente se ordenará su remoción o modificación en un plazo de (3) tres días hábiles. Las multas para los infractores incurrirán por la publicidad irregular de avisos, pendones, carteleras en una sanción de 15 salarios mínimos diarios legales vigentes, y en el caso de las vallas, 5 salarios mínimos mensuales vigentes.

En el año 2009 el Acuerdo 018 modifica la oficina de enlucimiento de fachadas, monumentos, descontaminación visual de la ciudad y limpieza del deterioro físico, ya que le posibilita desarrollar funciones que permiten recuperar y conservar las zonas y/o tipologías, espacios o sitios de interés en el municipio, que por sus características especiales constituyen patrimonio histórico, arquitectónico y ecológico de la ciudad, para eliminar la contaminación visual existente en la zona centro, mediante desmonte y supresión de los avisos y letreros en las edificaciones de acuerdo con lo establecido en el Acuerdo 028 de 2008.

En marzo de 2012, con el Acuerdo 084, se modifican el Acuerdo 041 de 2008 y el Acuerdo 066 de 2010, compilados en el Decreto 514 de 2011, para lograr la generación de incentivos tributarios transitorios durante los años gravables 2012, 2013, 2014 y 2015 a los contribuyentes que paguen por anticipado el impuesto de industria y comercio, avisos y tableros, las sobretasas bomberil y sobretasa con destino al deporte, la recreación y el aprovechamiento del tiempo libre.

Es en el año 2013 por medio del Acuerdo 5361, cuando se prohíbe el ingreso de toda clase de vendedores ambulantes a unas áreas determinadas de la ciudad de Sincelejo, como estrategia contemplada en el Plan de Desarrollo Municipal 2012 - 2015, para construir las ciudades amables de la visión colombiana 2019.

\section{CONCLUSIONES Y RECOMENDACIONES}

\section{Crisis de la ciudadanía, crisis de la ciudad.}

Las versiones descritas de contaminación visual por tratadistas en el tema y las versiones expuestas por los ciudadanos coinciden en su gran mayoría, lo que indica la existencia de un conocimiento conceptual inclusive de los actores de estos impactos, ultimando su discurso con las afectaciones que este tipo de contaminación hace, no solo sobre el paisaje o el elemento físico, sino sobre la valoración del sujeto y las molestias que estos causan al mismo.

Los tipos de contaminación identificados y que se hacen presentes en su mayoría en la ciudad de Sincelejo, afectan directamente sobre los bienes de características históricas de este centro urbano, disminuyen su valor patrimonial e inciden negativamente a la importante valoración que se debe tener de ellos tanto por los oriundos de la ciudad como por los visitantes turistas.

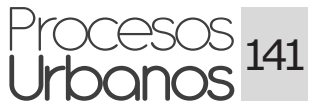


Esta disminución de la valoración de la estética del paisaje urbano del centro histórico de la ciudad afecta considerablemente la legitimización de los espacios y por ende, la competitividad urbana a nivel regional y nacional.

Ante la presencia inevitable de la contaminación visual en la ciudad de Sincelejo, se hace necesario no solo la aplicación y cumplimiento estricto de las normas y leyes que permitan regular este hecho, sino, también es importante la divulgación de ellas al público en general, como un modelo pedagógico y de participación, de manera que se convierta en un constructor de los valores ciudadanos. Desarrollar una propuesta de un plan estratégico de descontaminación visual, que implica promover el reconocimiento de los elementos estructurantes del espacio público del centro de la ciudad de Sincelejo y la valoración de las características arquitectónicas y urbanas de conservación histórica que lo representan, catalogando las afectaciones que este tipo de contaminación ambiental causa en el bienestar y confort ciudadano, en relación con la imagen de la ciudad, de manera que se convierta en una herramienta para intervenir el espacio físico afectado, a través de líneas y criterios de acción y participación.

\section{REFERENCIAS}

Acuerdo 28 de 2008. Sincelejo - Sucre.

Díaz Araujo, María M. (1997) Daño por contaminación ambiental urbana. Polución, impacto auditivo, visual y ambiental, Ed - 1997 - IV - Pág. 686.

Fernández, D. E. (2003) La contaminación visual en la vía pública. Trabajo de tesis de Licenciatura en Diseño Gráfico. Universidad Abierta interamericana. Buenos Aires, Argentina. Recuperado de: http://imgbiblio.vaneduc.edu.ar/fulltext/files/TC048684.pdf acceso 13 de octubre de 2016.

Gámez, E. M. (2013) La contaminación visual actual de los avisos publicitarios en la ciudad de Bogotá. Universidad Militar de Colombia, Facultad de Ciencias Económicas. Especialización en Mercadeo de Servicios. Seminario de grado. Recuperado de: http://repository.unimilitar.edu. co/bitstream/10654/10716/1/ELSA\%20GAMEZ.pdf acceso octubre 12 de 2016.

García M. (2013). La contaminación visual. Recuperado de: http://www.virtual.unal.edu.co/ cursos/ciencias/mtria ensenanza/form int/und 1/pdf/ambiente/la contaminacion visual. pdf acceso, octubre 10 de 2016.

Méndez, V. (2013). La contaminación visual en espacios públicos de Venezuela. Revista Gestión y Ambiente. Vol. 16 (no. 1). Medellín.

Plan de desarrollo municipal 2012 - 2015. Sincelejo - Sucre. Alcaldía municipal de Sincelejo. Rapoport, A. (1974). Aspectos de la calidad del entorno. Barcelona: la Gaya Ciencia, S.A. 